\title{
Composite multiferroic materials consisting of NdFeB and PZT particles embedded in elastic matrix: the appearance of electrical polarization in a constant magnetic field
}

\author{
Liudmila Makarova1,, ${ }^{1,}$ Yuliya Alekhina ${ }^{1}$,Elena Kramarenko $^{1}$, Alexander Omelyanchik ${ }^{2,3}$, Valeria Rodionova ${ }^{2,3}$, \\ Olga Malyshkina ${ }^{4}$ and Nikolai Perov ${ }^{1,2}$ \\ ${ }^{1}$ Lomonosov Moscow State University, Faculty of Physics, 119991Moscow, Leninskie gory 1-2, Russia \\ ${ }^{2}$ Immanuel Kant Baltic Federal University, 236004 Kaliningrad, Nevskogo 14, Russia \\ ${ }^{3}$ Center for Functionalized Magnetic Materials (FunMagMa), Immanuel Kant Baltic Federal University, 236041, Kaliningrad, Russia \\ ${ }^{4}$ Tver State University, 170100, Tver, Russia
}

\begin{abstract}
New composite materials consisting of polymer matrix with PZT and NdFeB microparticles were prepared and investigated in the work. It was found that magnetic properties such as saturation magnetization, coercivity, permeability, depend on mass concentration of the ferromagnetic particles in the samples. Also it was found that all samples had electrical polarization in DC external electric field. The electric properties such as coercivity, remanent polarization, the maximum polarization value, had changes in the external constant magnetic field $1.1 \mathrm{kOe}$. These changes depended on both concentrations of ferromagnetic and ferroelectric particles. This type of magnetoelectric transformation allows us to classify new materials as multiferroic materials. These new composite materials can easily be prepared of any shape, the final materials are flexible and resistant to external chemical influences. The area of application of new multiferroic materials varies from sensors to autonomous energy sources.
\end{abstract}

\section{Introduction}

Smart materials are materials which convert one form of energy to another [1]. Usual ferromagnetic or ferroelectric materials are also smart. Magnetostrictive effect which is observed in ferromagnetic materials is the example of changes of shape and sizes in the external magnetic field. Piezoelectric, magnetostrictive and ferroelastic materials have been already used in sensors, actuators and controllers. The area of application of smart materials includes different types of sensors, microphones, injectors, energy harvesting and well logging [2,3]. Multiferroic materials are smart structures due to combination of magnetostriction and piezoelectric properties in one material $[4,5]$.

Magnetorheological materials such as fluids, foams, elastomers are smart structures. Well known examples of energy transformation are magnetorheological effect, magnetodeformation effect, Payne effect, magnetodielectric effect $[6,7]$.

New type of composite multifunctional material with both ferromagnetic, ferroelectric and ferroelastic properties was prepared. Unlike the existing types of composite multiferroics, such materials are easy to prepare, they are flexible and chemoresistive.

Previously it has been shown that magnetodielectric effect was observed in magnetic elastomers, namely the dielectric permittivity increase in the external magnetic field [8]. It is supposed that magnetic moments of all ferromagnetic particles rotate to the field direction and particles tend to shift forming chain-like structures. The internal stresses arise in the elastic matrix and these stresses can result in rotation or displacement of ferroelectric particles or prevent it what results in the changes of electric properties. In this work we investigated the influence of external magnetic field on the electric properties of new three-component composite material. Also we investigated the effect of ferroelectric particles on the magnetic properties of the samples. The direct magnetoelectric transformation in new three-component samples was found. The opposite effect can be observed: the displacement of ferroelectric particles leads to changes in magnetic properties [9].

\section{Materials and methods}

\subsection{Materials}

The preparation of ferromagnetic and ferroelectric particles was described in our previous work [9]. Filling particles were hard magnetic $\mathrm{NdFeB}$ particles (sizes varied from 5 to $30 \mu \mathrm{m}$ ) and ferroelectric particles PZT26 (size was about $5 \mu \mathrm{m}$ ). There were prepared 7 samples, based on silicone polymer, the sample compositions are presented in Table 1. The components of polymer compound with total volume $1 \mathrm{ml}$ were mixed with powders. The amount of each type of powder was calculated from mass concentration and density of each material. The correlations between volume and mass concentration are also presented in Table 1. The total volume concentrations of filling powder varied from $20 \%$ to $40 \%$. The volume ratios between $\mathrm{NdFeB}$ and PZT particles were $1: 1,2: 1,1: 3$ and $1: 6$. Also the elastomer with $20 \mathrm{vol} \%$ of PZT particles was prepared.

\footnotetext{
*Corresponding author: la.loginova@physics.msu.ru
} 
Table 1. Samples composition.

\begin{tabular}{|c|c|c|c|c|}
\hline $\begin{array}{c}\text { Sample } \\
\text { No. }\end{array}$ & $\begin{array}{c}\text { NdFeB:PZT } \\
\text { (vol) }\end{array}$ & $\begin{array}{c}\text { Total } \\
\text { vol. \% }\end{array}$ & $\begin{array}{c}\text { NdFeB } \\
\text { mass \% }\end{array}$ & $\begin{array}{c}\text { PZT } \\
\text { mass \% }\end{array}$ \\
\hline $0-1$ & $0: 1$ & 20 & - & 50.5 \\
\hline 1 & $1: 6$ & 20 & 12.0 & 41.0 \\
\hline 2 & $1: 3$ & 20 & 20.3 & 34.5 \\
\hline 3 & $1: 1$ & 20 & 37.3 & 21.2 \\
\hline 4 & $2: 1$ & 20 & 47.2 & 13.4 \\
\hline 5 & $1: 1$ & 30 & 45.1 & 25.6 \\
\hline 6 & $1: 1$ & 40 & 50.4 & 28.6 \\
\hline
\end{tabular}

\subsection{Methods}

Magnetic properties were investigated by vibrating sample magnetometer (VSM) Lake Shore 7400 in the field range $16 \mathrm{kOe}$ at room temperature. The sample for measurement with sizes $3 \mathrm{~mm} * 3 \mathrm{~mm} * 1 \mathrm{~mm}$ was cut from the material and fixed at the holder, the plane of the sample was parallel to the direction of the external magnetic field.

Also using VSM the magnetic relaxation properties were investigated. The time dependences of the magnetic moment of the sample were measured either with switching on the external magnetic field or after switching off the external magnetic field. The sample was placed between the copper plates (using a nonconductive gasket between the samples and the plate to avoid accidental breakdown currents) and fixed at the holder at the same orientation (plane was parallel to the direction of magnetic field). Plates were connected to the high voltage source. Magnetic relaxation properties were also measured with switching on the external electric field with the voltage $5 \mathrm{kV}$.

The electric properties, namely the dependence of electric polarization on the electric field, were investigated by Soyer-Tauer method. The ferroelectric hysteresis loop was observed at the screen of the oscilloscope. The AC voltage from the generator is transmitted to the series-connected sample and the reference capacitor. The input $\mathrm{Y}$ of the oscilloscope is supplied with a voltage from the reference capacitor. The input $\mathrm{X}$ is supplied with a voltage from the ferroelectric sample.

The sample with average sizes $1.5 \mathrm{~cm} * 1.5 \mathrm{~cm} * 2.5 \mathrm{~mm}$ was covered by liquid silver solution at both sides and placed between conducting clips. The polarization $P$ of the sample is calculated from the formula:

$$
P=\frac{C_{r e f} * U_{\text {out }}}{S}
$$

Where $C_{\text {ref }}=1 \mu \mathrm{F}$ - reference capacitor, $U_{\text {out }}$ - value of voltage of output signal (ordinate axes at the screen of oscilloscope), $S$ - square of the sample's electrodes.

The electric field $E=U^{*} d$, where $U$ - the amplitude of AC voltage (abscissa axes at the screen of oscilloscope), $\mathrm{d}$ - the distance between the plates with the sample, or the width of the sample.

The influence of external magnetic field on the electric properties was measured by placing the sample into the magnetic field of permanent calibrated magnet. The plane of the sample was perpendicular to the direction of magnetic field.
Phase transition properties were investigated with Differential Scanning Calorimeter NETZSCH 204 F1 Phoenix at the temperature range from $0 \mathrm{C}$ to $450 \mathrm{C}$.

\section{Results and discussion}

\subsection{Magnetic properties}

The typical hysteresis loops of different samples are presented in Figure 1.

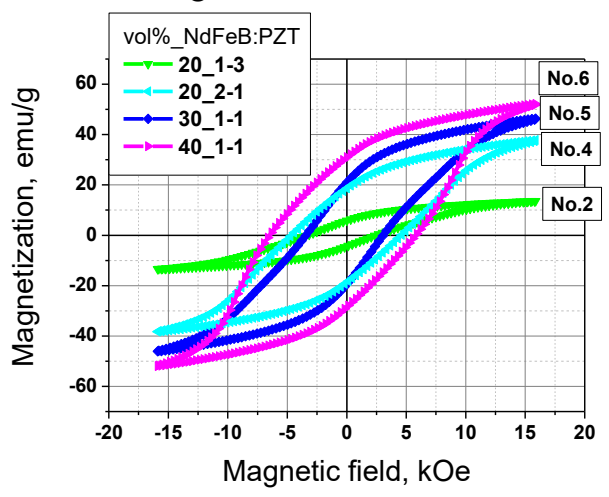

Fig. 1. The dependences of magnetization of the samples 2, 4, 5,6 on magnetic field at room temperature.

All magnetic parameters such as maximum magnetization, coercivity, remanent magnetization depend on the sample composition. For example maximum magnetization increases with the increase of mass concentration of ferromagnetic particles, what is presented in Figure 2. As the saturating field of $\mathrm{NdFeB}$ material is about $25-30 \mathrm{kOe}$, we can not present the dependence of saturation magnetization on concentration of magnetic filler. In the field $16 \mathrm{kOe}$ not all magnetic moments of the particles can be oriented in the direction of the external magnetic field. Moreover, in nonsaturating field it is necessary to take the interaction between magnetic particles and the change of the internal field associated with this interaction into account. Therefore, the Figure 2 presents the nonlinear dependence of magnetization in the maximum field on the concentration of magnetic filler.

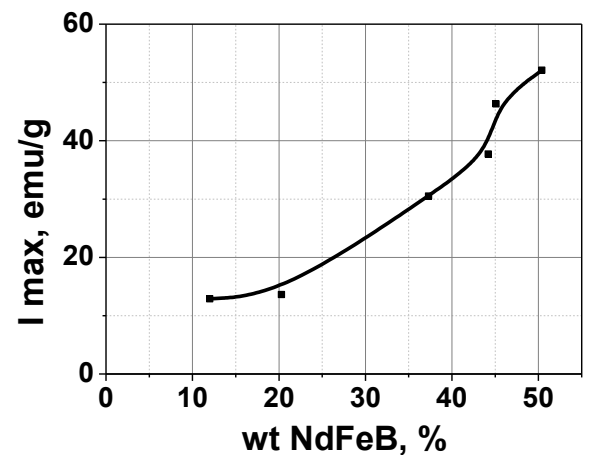

Fig.2.The dependence of maximum value of magnetization of the samples 1-6 on mass concentration of $\mathrm{NdFeB}$ particles. 
The elastic properties depend on the relative concentration of PZT particles. Namely the Young's modulus decrease with increase of relative content of PZT particles (Fig.3).

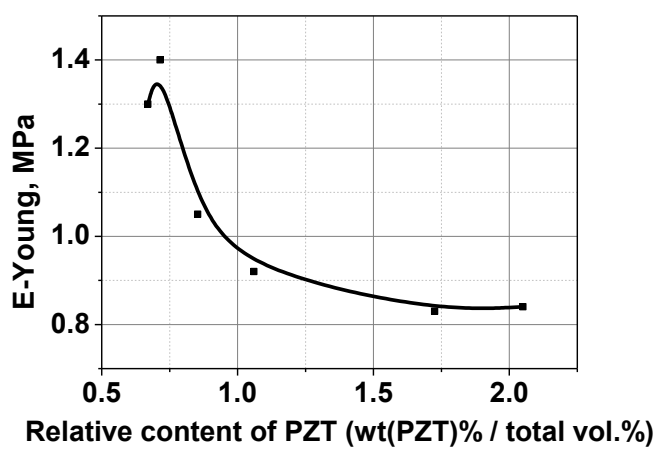

Fig.3.The dependence of Young's modulus of the samples 1-6 on the relative concentration of PZT particles.

The dependence of Young's modulus on PZT particles concentration is significant as the coercivity depends on elastic modulus (Fig.4a). It was shown earlier [10] that the remagnetization of the sample is associated with the rotation of the ferromagnetic particle in the elastic medium. In softer medium the elastic force acting on the particle is smaller, so the coercivity has a smaller value.

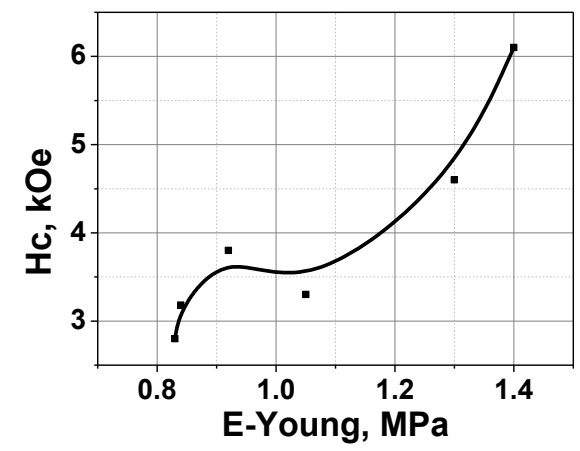

Fig.4(a).The dependence of coercivities of the samples 1-6 on the corresponding Young's modulus.

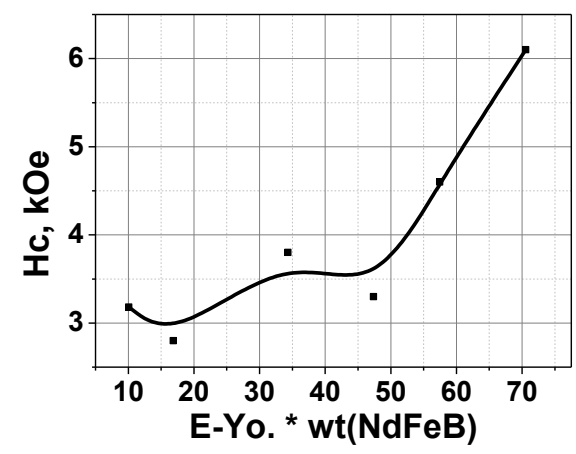

Fig.4(b).The dependence of coercivities of the samples 1-6 on both Young's modulus and mass concentration of NdFeB particles.
At the same time coercivity depends on concentration of ferromagnetic particles as interparticle interactions influence on the remagnetization process. The dependence of coercivity on both elastic properties and concentration of $\mathrm{NdFeB}$ particles is presented in Figure 4(b).

\subsection{Ferroelectric properties}

The dependences of polarization on electric field were measured for each sample with ferroelectric particles, including the sample with only ferroelectric particles No.0-1.

Also temperature measurements were carried out with Differential Scanning Calorimeter NETZSCH 204 F1 Phoenix. Curie temperature of PZT powder was 321.1 C. All electric measurements were carried out at room temperature, so we assume that samples revealed ferroelectric hysteresis properties.

It was confirmed that addition of PZT particles to the silicone matrix led to appearance of ferroelectric properties of the material (Fig. 5-10). For example, the dependence of electric polarization of the sample No.0-1 with PZT particles on electric field at frequencies $20 \mathrm{~Hz}$ and $50 \mathrm{~Hz}$ are presented in Figure 5. Moreover the loop at lower frequency $20 \mathrm{~Hz}$ has larger value of coercivity than the loop at higher frequency $50 \mathrm{~Hz}$. It should be noted that at even higher frequencies $(100 \mathrm{~Hz}$ for instance) the loop transformed to straight line. The loop is not saturated for several reasons: 1) the value of saturating electric field exceeds $1 \mathrm{kV} / \mathrm{mm}$, that can not be achieved at the equipment; 2) the width of the sample was about $2 \mathrm{~mm}$, that decreases the total value of electric field; using of thinner sample may lead to breakdown of capacitor.

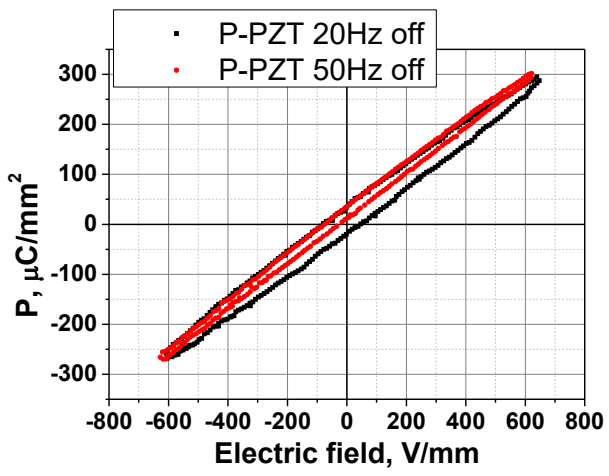

Fig.5.The dependences of polarization of the sample No.0-1 on electric field at different frequencies.

It was found that hysteresis loops of different samples were observed at different modes of generator - at different frequencies and at different amplitudes of the electric field. In Figure 6 the hysteresis loops of the samples No. 3, 5, 6 are presented. These loops were obtained at the extreme mode of generator, namely, at lower frequency or at the lower value of amplitude the loop was not observed. 


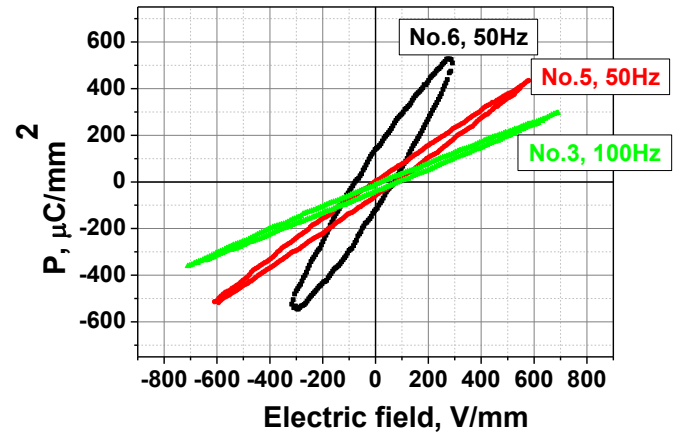

Fig.6. The dependences of polarization of the samples 3, 5, 6 on electric field.

Electric properties changed in the external magnetic field. The maximum value of polarization and coercive electric field increased in the magnetic field. For example, the change of the electric hysteresis loop of the sample No.6 in the external magnetic field is presented in Figure 7.

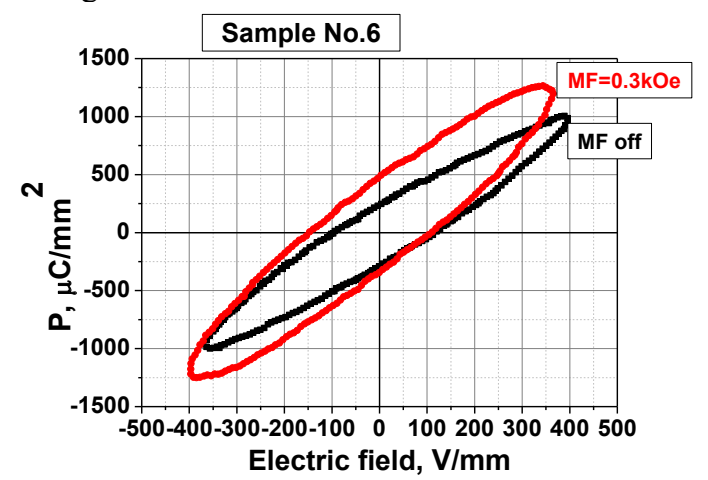

Fig.7.The dependences of polarization of the sample No.6 on the electric field in zero magnetic field (black) and in $300 \mathrm{Oe}$ magnetic field (red).

The change of maximum value of polarization was about $25 \%$ for the sample No.6. Also the electric coercivity increased in magnetic field at $22 \mathrm{~V} / \mathrm{mm}$.

The coefficient of magnetoelectric transformation is the change of electric coercivity divided by value of magnetic field $\left(\alpha_{\mathrm{E}}=\Delta \mathrm{Ec} / \mathrm{H}, \mathrm{V} /(\mathrm{mm} * \mathrm{Oe})\right)$, so the values of this coefficient were calculated for all samples (Table 2). It was not found any dependence of the coefficient $\alpha_{\mathrm{E}}$ on the elastic, ferroelectric or ferromagnetic characteristics of materials.

Table 2. Young's modulus and the coefficient of electric transformation for the samples 1-6.

\begin{tabular}{|c|c|c|}
\hline $\begin{array}{c}\text { Sample No., } \\
\text { (vol\%_NdFeB:PZT) }\end{array}$ & $\begin{array}{c}\text { E-Young, } \\
\mathrm{MPa}\end{array}$ & $\begin{array}{c}\alpha_{\mathrm{E}}=\Delta \mathrm{Ec} / \mathrm{H}, \\
\mathrm{V} /(\mathrm{cm} * \mathrm{Oe})\end{array}$ \\
\hline $1\left(20 \_1: 6\right)$ & 0.84 & 0.14 \\
\hline $2\left(20 \_1: 3\right)$ & 0.83 & 0.43 \\
\hline $3\left(20 \_1: 1\right)$ & 0.92 & 0.19 \\
\hline $4\left(20 \_2: 1\right)$ & 1.3 & 0.11 \\
\hline $5\left(30 \_1: 1\right)$ & 1.05 & 0.44 \\
\hline $6\left(40 \_1: 1\right)$ & 1.4 & 0.73 \\
\hline
\end{tabular}

Also the anisotropy of the samples was found. Namely, the placing of the sample by different sides to the magnet leaded to different changes in the hysteresis loop. Firstly the hysteresis loop was measured for the sample without magnetic field, then the sample was placed by one side to the magnet (first direction), then the sample was turned by $180^{\circ}$ and was placed by another side to the magnet (second direction). In Figure 8 the results of initial loop and loops in the magnetic field are presented.

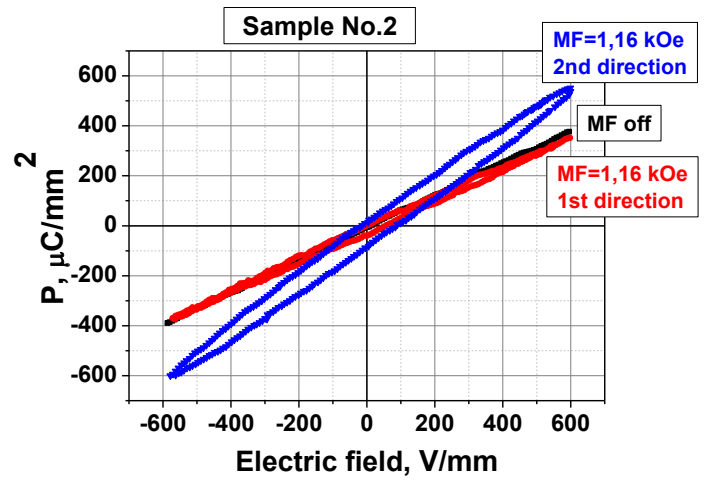

Fig.8.The dependences of polarization of the sample No.2 on electric field in zero magnetic field (black), in 1.16kOe magnetic field applied in first direction (red), in $1.16 \mathrm{kOe}$ magnetic field applied in second direction (blue).

If the sample was placed to the magnet by first side, the changes of electric properties were not observed. And the turning of the sample to the other side leaded to significant changes in the electric properties. This anisotropy could appear from the previous measurements in the external magnetic field [9]. This anisotropy is associated with the alignment of magnetic moments of magnetic particles. In the first case the value of magnetic field is too small, the direction on magnetic field is opposite to the direction of magnetic moments of the particles, and particles stay at their position. In the second case the particles can move in the elastic matrix, producing the internal stresses in the matrix, that can change the electric polarization of the sample.

At the smaller concentration of ferroelectric particles, the electric hysteresis loop was almost not observed (Figure 9, sample No.1). The electric hysteresis loop of the sample No.1 was not observed even in the external magnetic field, but after taking the sample out from the magnetic field, the explicit hysteresis loop appeared.

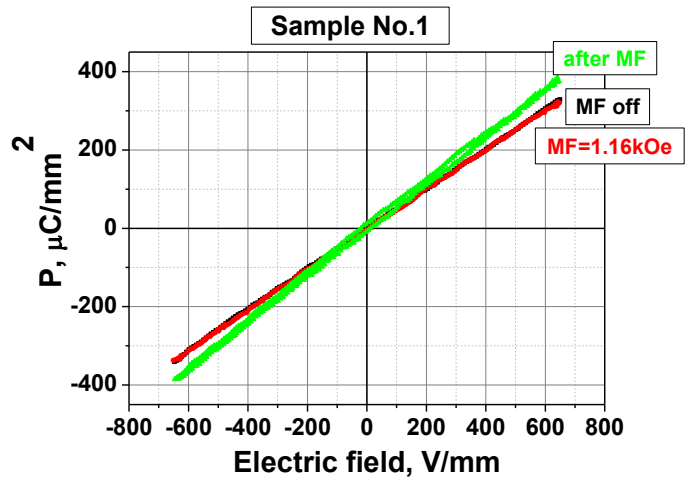

Fig.9.The dependences of polarization of the sample No.1 on electric field. 
The other sample (No.5) showed the smooth decrease of the electrical parameters of the loop after the magnetic field switched off, which is presented in Figure 10(a,b). It is probably associated with magnetic relaxation properties of the samples.
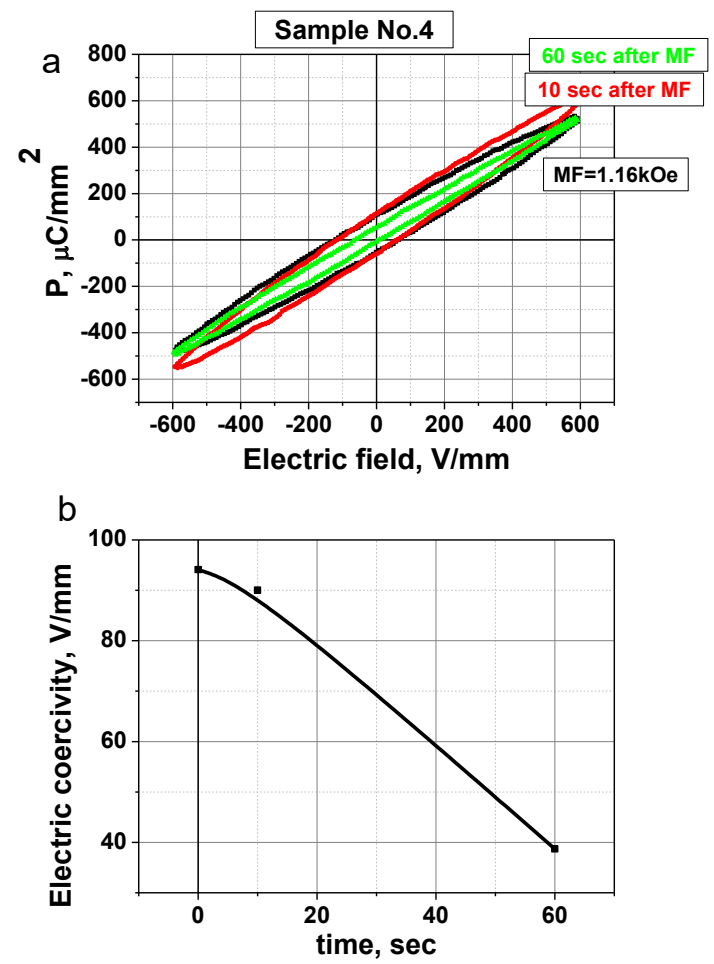

Fig.10(a,b).a) the dependences of polarization of the sample No.5 on electric field in magnetic field (black), 10 seconds after magnetic field switched off (red), 60 secondsafter magnetic field switched off (green); b) the dependence of electric coercivity of the sample No.5 on time after magnetic field switched off.

\subsection{Magnetic relaxation properties with external electric field}

The magnetic relaxation properties were measured in two modes: first mode was in the constant magnetic field $500 \mathrm{Oe}$, second mode was after switching magnetic field off. For example, the relaxation time dependence for the sample No.6 is presented in Figure 11 [9]. The relaxation time $\tau$ for each section was determined by approximating by the exponential function $e^{ \pm t / \tau}$. The relaxation time in the first mode in the external electric field exceeds one without electric field. We proposed that the internal stresses, induced by displacements of ferroelectric particles in external electric field, prevent the rotation of ferromagnetic particles in the field direction. The relaxation times approximately coincide after the magnetic field is turned off (second mode).

Also it was found that it takes a long time to reach the constant value of magnetic moment after switching the magnetic field off. This magnetic relaxation influences the electric properties of the materials, namely, the dependence of electric coercivity on time after magnetic field is switched off (Fig.10b).

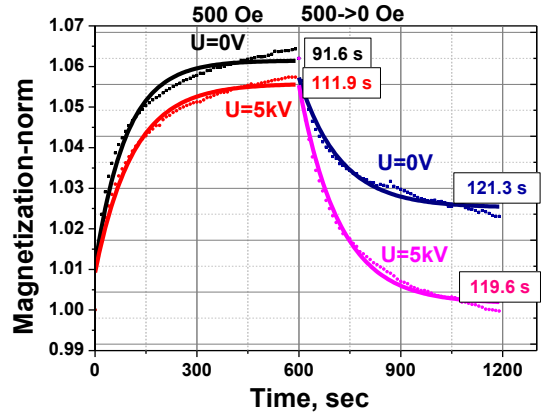

Fig.11.The dependence of normalized magnetization of the sample No.6 on time in different modes in zero and $5 \mathrm{kV}$ external electric voltage [9].

\section{Conclusion}

New composite materials with ferromagnetic, ferroelectric and ferroelastic properties were investigated in the work. It was found that ferroelectric particles influence the Young's modulus of the composite, indirectly affect the magnetic characteristics (coercivity). It was shown that materials change their electric properties in the external magnetic field. Maximum value of the coefficient of magnetoelectric transformation is $0.73 \mathrm{~V} /(\mathrm{cm} * \mathrm{Oe})$. Magnetic relaxation properties were investigated and it was shown that magnetic relaxation influences the electric properties of the materials.

\section{Acknowledgement}

This work was supported by the Ministry of Education and Science of the Russian Federation in the framework of government assignments 3.9002.2017/6.7 and 3.4168.2017/4.6

\section{References}

1. Y. Huang, et.al., Adv. Mat., 8344-8364 (2016)

2. R. Sabo, et.al., J. of Renewable Mat., 4, 297 (2016)

3. Y. Yang, et.al., Mat. Sc. And Engineering R: Reports, 115, 1-37 (2017)

4. Q. Zhu, et.al., J. Mater. Res., 29, 657 (2014)

5. J. Zhang, et.al., Sensors and Actuators A, 214, 149 (2014)

6. Y. Li, et.al., Smart Materials and Structures, 23 (12), 123001 (2014)

7. X.L. Gong, et.al., Int. J. Mod. Phys. B. 21 (28-29), 4875-7882 (2007)

8. A.S. Semisalova, et.al., Soft Matter, 9(47), 11318 (2013)

9. L.A. Makarova, et.al, IEEE Transactions on Magnetics, 53(11), 17-03-0349 (2017)

10. L.A. Makarova, et.al., JMMM, 440, 10-32 (2017). 